phys. stat. sol. (b) 234, No. 1, 139-146 (2002)

\title{
Photonic Bands and Radiation Losses in Photonic Crystal Waveguides
}

\author{
Lucio Claudio ANDREani ${ }^{1}$ ) \\ Istituto Nazionale per la Fisica della Materia (INFM) and Dipartimento \\ di Fisica "Alessandro Volta”, Università degli Studi di Pavia, via Bassi 6, 27100 Pavia, Italy
}

(Received September 1, 2002; accepted September 3, 2002)

PACS: 42.70.Qs; 78.67.-n

Dedicated to Professor Dr. Roland Zimmermann on the occasion of his 60th birthday

\begin{abstract}
A method for the theoretical description of two-dimensional (2D) photonic crystals embedded in planar waveguides is presented, which is based on an expansion of the magnetic field in the basis of guided modes of an effective waveguide. The method yields the dispersion of photonic modes below and above the light line, and in the latter case it gives also the radiative losses of quasi-guided modes due to diffraction out of the plane. Photonic bands and gap maps of strong- and weak-index contrast systems display confinement effects and other notable differences compared to the ideal 2D case. The radiative losses in periodic $2 \mathrm{D}$ systems are found to increase with the refractive index contrast between core and cladding, and with the radius of the holes in the triangular lattice.
\end{abstract}

1. Introduction Photonic crystals, namely materials with a periodic dielectric constant, were first proposed by Yablonovitch [1] and John [2] as a way to suppress spontaneous emission and to achieve localization of light by disorder. A periodic modulation of the refractive index leads to the validity of Bloch-Floquet theorem and to the formation of photonic bands, in analogy to electron bands in crystalline solids. A frequency region where no photonic energies are found and therefore light propagation cannot take place is called a photonic gap. A complete band gap occurs when light cannot propagate in any direction of the Brillouin zone, either for a selected polarization state or for both polarizations. After an initial period of slow development, the research field of photonic crystals has progressed very rapidly in recent years, especially when nanotechnology techniques for the fabrication of photonic structures in the near-infrared and visible regions have become available.

Three-dimensional photonic crystals are the only ones which may possess a truly complete band gap [3, 4], yet their fabrication at sub-micrometric scales is hard to achieve and not flexible enough to allow for the controlled introduction of line and point defects. Two-dimensional photonic structures embedded in planar waveguides, also known as photonic crystal slabs, can instead be fabricated in the near-infrared and visible regions by nanolithography and etching techniques [5-16]. In these systems, the propagation of light is controlled in the $2 \mathrm{D}$ plane by the photonic structure, and in the vertical direction by the dielectric discontinuity of the slab waveguide. Photonic crystal slabs, however, are characterized by the so-called light-line problem: only photonic modes which lie below the light line of the cladding material are truly guided and stationary, while modes lying above the light line are subject to radiation losses and are called quasi-guided modes, or guided resonances. Radiation losses of these modes corre-

1) e-mail: andreani@fisicavolta.unipv.it 
spond physically to out-of-plane diffraction of a Bloch wave which propagates in the plane; the issue of out-of-plane losses is a crucial one for prospective applications of photonic crystals to various kinds of devices.

While the energies of guided modes can be calculated by introducing a supercell in the vertical direction and using 3D plane-wave expansion [7], the frequency dispersion and especially the losses (i.e. the imaginary part of the frequency) of quasi-guided modes are more difficult to obtain and are often calculated by numerical simulations with the finite-difference time domain (FDTD) technique [14, 15]. Recently, another approach to the complex energies of quasi-guided modes based on finding the poles of a scattering matrix operator has been proposed $[17,18]$. In the present work we describe an alternative method which allows to calculate the energies of photonic modes in photonic crystal slabs, both below and above the light line; for quasi-guided modes, both real and imaginary parts of the energies are obtained. A few illustrative results are presented and discussed. More detailed results for the real part of the photonic mode dispersion can be found in Ref. [19]. All examples shown here refer to the triangular lattice of circular air holes as a photonic pattern.

2. Method To illustrate the method, let us start from the second-order equation for the magnetic field

$$
\nabla \times\left[\frac{1}{\epsilon(\mathbf{r})} \nabla \times \mathbf{H}\right]=\frac{\omega^{2}}{c^{2}} \mathbf{H},
$$

where $\epsilon(\mathbf{r})$ is the spatially-dependent dielectric constant. If the magnetic field is expanded in an orthonormal set of basis states labelled by the index $\mu$ as

$$
\mathbf{H}(\mathbf{r})=\sum_{\mu} c_{\mu} \mathbf{H}_{\mu}(\mathbf{r})
$$

then Eq. (1) is transformed into a linear eigenvalue problem

$$
\sum_{\nu} \mathcal{H}_{\mu \nu} c_{\nu}=\frac{\omega^{2}}{c^{2}} c_{\mu},
$$

where the "Hamiltonian" matrix $\mathcal{H}_{\mu \nu}$ is given by

$$
\mathcal{H}_{\mu \nu}=\int \frac{1}{\epsilon(\mathbf{r})}\left(\nabla \times \mathbf{H}_{\mu}^{*}(\mathbf{r})\right) \cdot\left(\nabla \times \mathbf{H}_{v}(\mathbf{r})\right) \mathrm{d} \mathbf{r} .
$$

In the present case of a photonic crystal slab we have a waveguide along $z$ and a periodic 2D patterning in the $x y$-plane. The basis set $\mathbf{H}_{\mu}(\mathbf{r})$ is chosen to consist of the guided modes of an effective waveguide, where each layer $j$ is taken to have a homogeneous dielectric constant given by the spatial average of $\epsilon_{j}(x, y)$ within the unit cell. The index $\mu$ of Bloch states can be written as $\mu=(\mathbf{k}+\mathbf{G}, \alpha)$, where $\mathbf{k}$ is the Bloch vector in the $x y$-plane, $\mathbf{G}$ is a $2 \mathrm{D}$ reciprocal lattice vector and $\alpha=1,2, \ldots, \alpha_{\max }$ is a discrete index which labels the guided modes at wavevector $\mathbf{k}+\mathbf{G}$. Both transverse electric (TE) and transverse magnetic (TM) guided modes are included in the expansion and are coupled by the dielectric modulation in the $x y$-plane. The matrix elements $\mathcal{H}_{\mu \mu^{\prime}} \equiv \mathcal{H}_{\mathbf{k}+\mathbf{G}, \alpha ; \mathbf{k}+\mathbf{G}^{\prime}, \alpha^{\prime}}$ of Eq. (4) can be calculated by noting that the $d x d y$ integral in each layer $j$ yields the Fourier transform $\epsilon_{j}^{-1}\left(\mathbf{G}, \mathbf{G}^{\prime}\right)$ of the inverse dielectric function, which is the same quantity which appears in $2 \mathrm{D}$ plane-wave calculations. 
The guided modes $\mathbf{H}_{\mu}(\mathbf{r})$ of the "effective" waveguide represent an orthonormal set of states, however, the basis set is not complete since the leaky waveguide modes are not included. When the guided modes are folded in the first Brillouin zone, most (sometimes all) of them fall above the light line, i.e. in the energy region of leaky modes. Coupling to leaky modes at all energies produces a second-order shift of the resonance energies of all modes: a comparison with exact reflectance calculations indicates that such a real energy shift is usually small [19]. More important, first-order coupling to leaky modes at the same energy leads to a radiative decay, i.e. to an imaginary part of the energy. This can be calculated by Fermi's golden rule, like in usual timedependent perturbation theory of quantum mechanics. The imaginary part of the frequency is given by

$$
-\operatorname{Im}\left(\frac{\omega_{k}^{2}}{c^{2}}\right)=\pi\left|\int \frac{1}{\epsilon(\mathbf{r})}\left(\nabla \times \mathbf{H}_{\text {leaky }}^{*}(\mathbf{r})\right) \cdot\left(\nabla \times \mathbf{H}_{\text {guided }}(\mathbf{r})\right) \mathrm{d} \mathbf{r}\right|^{2} \rho\left(\mathbf{k} ; \frac{\omega_{k}^{2}}{c^{2}}\right),
$$

where the $1 \mathrm{D}$ photonic density of states at fixed in-plane wavevector is [16]

$$
\rho\left(\mathbf{k} ; \frac{\omega_{k}^{2}}{c^{2}}\right)=\sum_{k_{z}>0} \delta\left(\frac{\omega^{2}}{c^{2}}-\frac{1}{\epsilon_{\text {clad }}}\left(\mathbf{k}^{2}+k_{z}^{2}\right)\right)=\frac{1}{4 \pi} \frac{n_{\text {clad }} c}{\sqrt{\omega^{2}-\frac{c^{2} \mathbf{k}^{2}}{\epsilon_{\text {clad }}}}}
$$

for each specified photonic mode polarization and parity. Only symmetric waveguides (i.e. with the same upper and lower claddings) are considered here. In this case, mirror symmetry $\sigma_{x y}$ with respect to a horizontal $x y$-plane bisecting the waveguide is a symmetry operation, and the solutions of Maxwell equations can be classified as even or odd with respect to specular reflection $\sigma_{x y}$.

3. Dispersion of Photonic Modes In Fig. 1a we show the dispersion of photonic modes in a membrane photonic crystal with air claddings, also called air bridge: the chosen value $\epsilon=12$ for the dielectric constant of the core corresponds to either Si or GaAs. The membrane is patterned with a 2D triangular lattice of holes. Such free-standing membranes have recently been fabricated and characterized [11, 12, 14]. A few modes lye below the cladding light line and are truly guided, due to the large refractive index contrast between the membrane material and the air, while all other modes lie above the light line and are quasi-guided. The dispersion of truly guided modes calculated by the present method is in good quantitative agreement with the results of 3D planewave calculations with a supercell in the vertical direction [7]. The dispersion of the even modes shows a gap between $a / \lambda=0.28$ and 0.37 : this is the well-known even gap of the triangular lattice of holes, and is shifted with respect to the gap in the ideal 2D case (which would occur between $a / \lambda=0.21$ and 0.27 for the assumed hole radius $r=0.3 a)$. The blue shift of mode energies in photonic crystal slabs with respect to the $2 \mathrm{D}$ case is a general phenomenon and is due to vertical confinement in the dielectric waveguide. In Fig. 1b we also show the dispersion of guided modes in the effective waveguide corresponding to the parameters of Fig. 1a, folded in the first Brillouin zone: it corresponds to the empty lattice dispersion in electronic structure calculations. The lowest (nonzero) energy level at the $\Gamma$ point is six-fold degenerate for both parities, due to the hexagonal symmetry of the photonic lattice. Comparison of Fig. 1a with Fig. 1b 

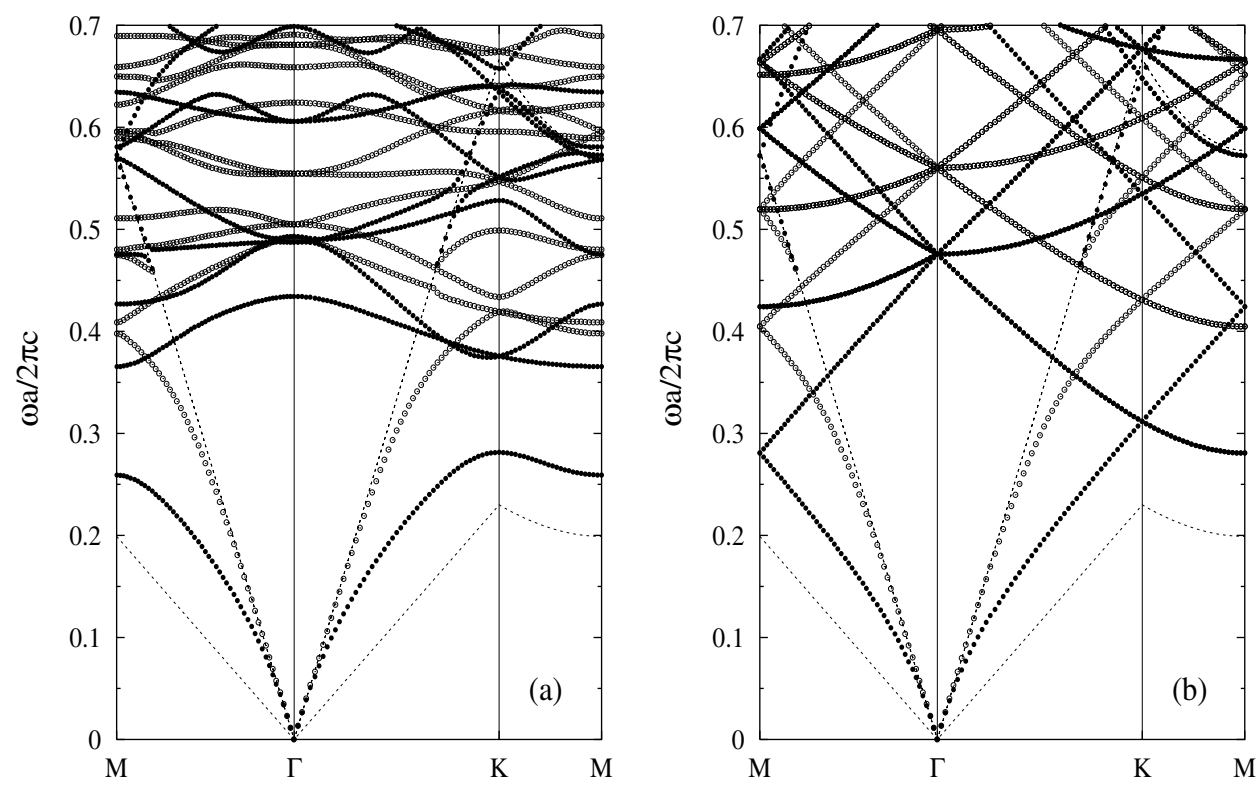

Fig. 1. Dispersion of photonic modes a) in a membrane photonic crystal slab with $\epsilon_{\text {core }}=12$, $\epsilon_{\text {clad }}=1$, and b) in the corresponding effective waveguide. The membrane is patterned with a triangular lattice of holes with pitch $a$ and radius $r=0.3 a$. The core thickness is $d=0.4 a$. Filled (open) circles: even (odd) modes with respect to specular reflection in the mid-plane of the waveguide. The dotted lines represent the dispersion of light in the effective core and cladding materials

indicates how band splittings and gaps occur when free waveguide photons are coupled by the off-diagonal Fourier components of the dielectric tensor. A second-order waveguide mode starts at $a / \lambda=0.46$ for both parities in the effective waveguide as well as in the patterned membrane. The cutoff energy of the second-order mode decreases on increasing the core thickness $(d=0.4 a$ in Fig. 1$)$.

Figure 2 shows the gap maps of the triangular lattice of holes in two photonic crystal waveguides with $\epsilon_{\text {core }}=12$ and $\epsilon_{\text {clad }}=1$ (Fig. 2a) or $\epsilon_{\text {clad }}=11$ (Fig. 2b): these values are representative of waveguides with a strong or weak refractive index contrast, respectively. The parameters of Fig. $2 \mathrm{~b}$ are close to those describing the GaAs/AlGaAs system. The core thickness is assumed to be $d=0.4 a$ : gap maps for other values of $d$ and for the ideal 2D case are given in Ref. [19]. Figure 2a shows that only an even gap occurs in the air bridge case for all values of the hole radius: the gap is blue-shifted with respect to the ideal 2D situation. In contrast, both even and odd gaps are found in Fig. $2 \mathrm{~b}$ for the GaAs/AlGaAs system, whose gap map is close to the ideal 2D one. The main difference for the gap map in a waveguide is that an overlap of even and odd gaps occurs for hole radii larger than about $0.31 a$ (compared to $0.4 a$ in the ideal 2D case): thus a complete band gap for all directions and polarizations opens already at relatively small values of the hole radius, which are easier to fabricate than lattices with small dielectric separation between the holes and are also more convenient in terms of propagation losses. 

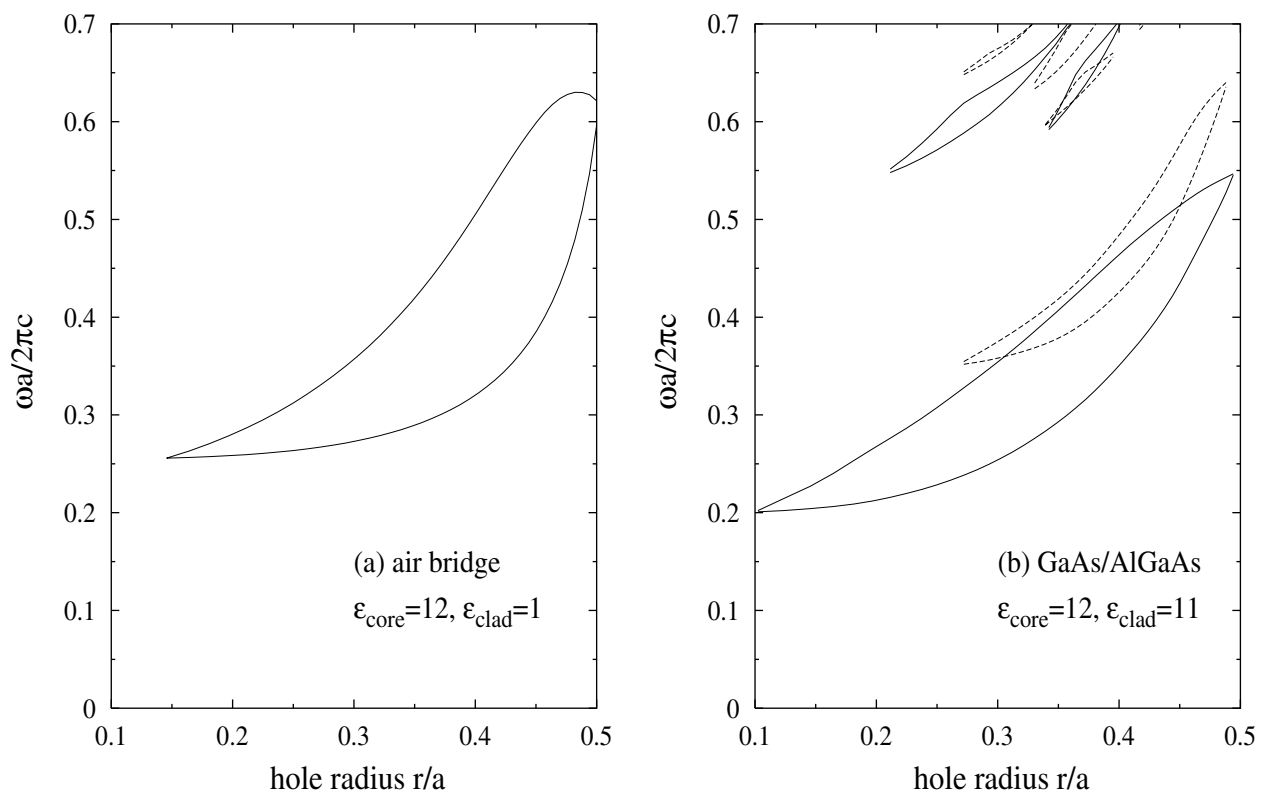

Fig. 2. Gap maps for the triangular lattice of air holes in photonic crystal waveguides with a) strong and b) weak refractive index contrast between core and cladding. The waveguides are patterned with a triangular lattice of pitch $a$ and variable hole radius. The core thickness is $d=0.4 a$ in both cases. Solid (dashed) lines represent the edges of photonic gaps for modes which are even (odd) with respect to specular reflection in the mid-plane of the waveguide

4. Diffraction Losses of Quasi-Guided Modes Figure 3 shows a 3D plot of the complex energy dispersion of even modes in a membrane photonic crystal with core thickness $d=0.3 a$ and hole radius $r=0.3 a$ of the triangular lattice. The $x y$-projection gives the real energy dispersion (similar to that of Fig. 1a), while the $z$-axis gives the imaginary part of the frequency. The losses are nonzero above the light line of the cladding material (air in this case), while they vanish for truly guided modes below the light line.

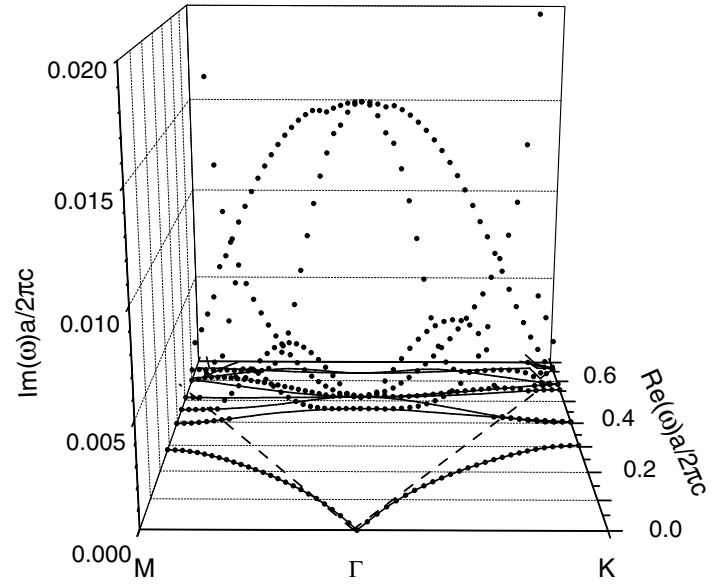

Fig. 3. Three-dimensional plot of the complex energies of photonic modes in a photonic crystal slab with $\epsilon_{\text {core }}=12$, $\epsilon_{\text {clad }}=1$. The membrane is patterned with a triangular lattice of holes of pitch $a$ and radius $r=0.3 a$. The core thickness is $d=0.3 a$. Solid curves represent the photonic mode dispersion (real part of the energy), while points represent the imaginary part ( $z$-axis) of the energy related to radiation losses. Only even modes are plotted. The dashes lines represent the light dispersion in air 
The imaginary part of the frequency is at most of the order of 0.02 , i.e. is small in absolute terms, thereby justifying the use of perturbation theory. $\operatorname{Im}(\omega)$ is different for each mode and depends strongly on the wavevector. The losses calculated by the present finite-basis expansion method are in good quantitative agreement with those obtained with exact FDTD simulations in three dimensions [15]: this validates the present approximation of treating coupling to leaky modes within time-dependent perturbation theory. For most of the modes, $\operatorname{Im}(\omega)$ grows on approaching the light line (reflecting the divergence of the 1D density of states (6)), however, it always goes to zero when crossing the light line: this is due to the behavior of the coupling matrix element, as shown in Ref. [16] within a perturbative treatment of the in-plane dielectric modulation. At the $\Gamma$ point, most of the modes are lossless, while only a two-fold degenerate mode has a finite imaginary part of the frequency: in fact only modes with the symmetry of the dipole can be coupled to the radiation field at normal incidence, while modes with different symmetries become radiative only at oblique incidence.

A mode above the light line with a finite $\operatorname{Im}(\omega)$ is subject to diffraction out of the plane during propagation; correspondingly, it can be excited by an external electromagnetic field incident on the surface of the photonic crystal slab. This is the basis for the surfacecoupling method proposed in Ref. [8] for the measurement of the photonic band dispersion in a waveguide: the reflectivity from the sample surface displays sharp resonant features which are associated with the excitation of photonic modes, and which yield the energy and the Bloch vector in the first Brillouin zone. Reflectance spectra at different angles of incidence and for different sample orientations allow to map the dispersion of quasi-guided modes in the whole Brillouin zone. The surface-coupling technique has been used by other groups $[20,21]$ and has become an important characterization technique, which is complementary to in-plane transmission in the waveguide. Most resonant features become vanishingly weak at normal incidence, when they correspond to modes which do not have dipolar symmetry at the $\Gamma$ point, while only the resonances corresponding to symmetry-allowed modes at $\Gamma$ remain strong at normal incidence [20].
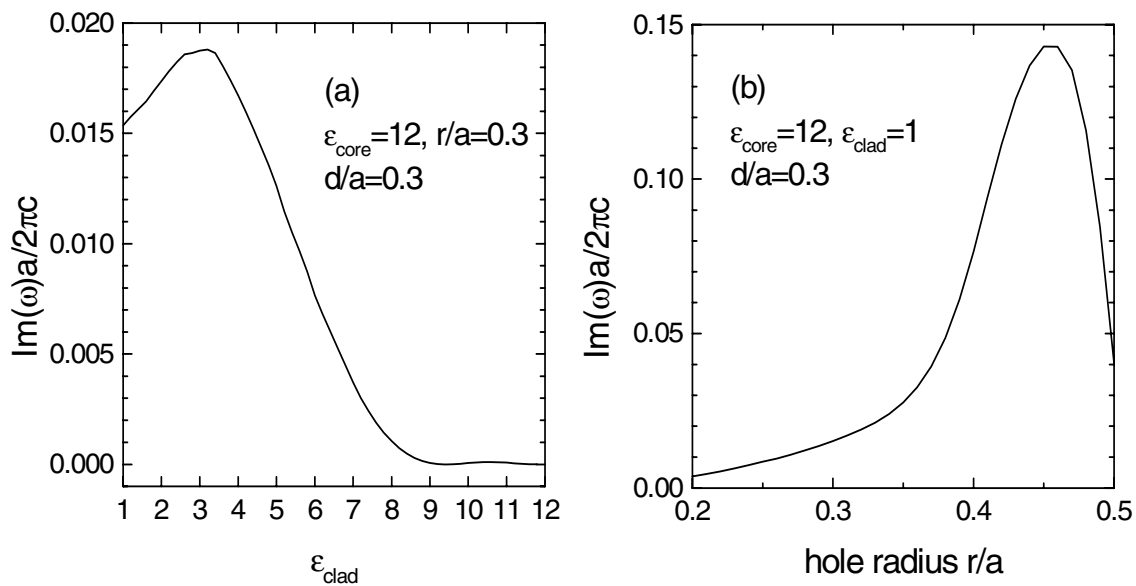

Fig. 4. Imaginary part of the energy for the lowest dipole-allowed even mode at the $\Gamma$ point in photonic crystal waveguides patterned with a triangular lattice of holes of pitch $a$, as a function of (a) the cladding dielectric constant, at fixed hole radius $r=0.3 a$, and (b) the hole radius, for $\epsilon_{\text {clad }}=1$. The core thickness is $d=0.3 a$ in both cases 
It is important to know how diffraction losses depend on various parameters, e.g. the dielectric contrast between core and cladding and the filling fraction of the 2D lattice. The radiative width of the dipole-allowed mode at the $\Gamma$ point can be used to this purpose, because it is a single number whose behavior can be studied as a function of structure parameters. Figure 4 shows $\operatorname{Im}(\omega)$ of the lowest symmetry-allowed mode at the $\Gamma$ point for different values of the refractive index contrast in the waveguide (Fig. $4 \mathrm{a}$ ) and of the hole radius (Fig. 4b) in the triangular lattice. In both cases the core thickness is $d=0.3 a$. Figure 4a indicates that $\operatorname{Im}(\omega)$ is much larger for a strong refractive index contrast in the vertical direction than in the case of a weak refractive index contrast. This is in agreement with a previous model [10], based on a zeroth-order separable approximation for the dielectric constant, which suggested that the diffraction losses scale as $(\Delta \epsilon)^{2} \equiv\left(\epsilon_{\text {core }}-\epsilon_{\text {clad }}\right)^{2}$. Figure $4 \mathrm{~b}$ shows that the losses increase rapidly with the air fraction in the triangular lattice: interestingly, they reach a maximum for $r=0.45 a$ and decrease again when the holes are enlarged towards the close-packing condition. These results show that a picture of radiation losses being localized in the air holes [10] is to a large extent correct for the triangular lattice, but still somewhat incomplete: rather, they suggest that the losses go to zero when either the air fraction or the dielectric fraction are made to vanish.

5. Conclusions The expansion in the basis of guided modes of an effective waveguide is seen to be an efficient and physically transparent method for the calculation of mode dispersion and diffraction losses in photonic crystal slabs. The method takes full account of the three-dimensional nature of the patterned waveguide, yet it is conceptually and formally close to a two-dimensional plane-wave calculation. The approximation of neglecting the leaky modes of the effective waveguide for the mode dispersion, and of treating them by Fermi's golden rule for the diffraction losses, is justified by comparison with results obtained with other more exact methods [7, 15].

The photonic mode dispersion in a photonic crystal slab displays confinement effects with respect to the ideal 2D case; within the present method, the mode dispersion can also be usefully compared with the dispersion of "free waveguide photons", which are the analog of free electrons or the empty lattice in the theory of electron bands in solids. In the specific case of gap maps for the triangular lattice of holes, results for the air bridge case show that only the even gap occurs (thus there is no complete band gap for both polarizations at any hole radius), while for the GaAs/AlGaAs system a full band gap for both polarizations may occur already for hole radii $r$ larger than about $0.3 a$. The occurrence of second-order or higher waveguide modes, which may spoil the presence of a gap, must be analyzed in any specific situation and favors the choice of small values for the core thickness, particularly for high index-contrast systems.

The diffraction losses of quasi-guided modes above the light line are found to be complicated functions of the mode index and of the wavevector. The imaginary part of the frequency often increases when the mode approaches the light line, but eventually goes to zero for all modes in the guided mode region. At $\mathbf{k}=0$, only modes with the symmetry of the dipole have finite losses due to diffraction in the normal direction. Looking at the trends as a function of structure parameters, the losses increase on increasing the dielectric contrast between core and cladding and on increasing the air fraction in the triangular lattice, with a maximum around $r / a=0.45$. These trends are expected to hold also for other geometries and for linear waveguides in photonic crys- 
tal slabs: in the latter case, specific calculations of $\operatorname{Im}(\omega)$ will yield propagation losses of defect modes. This will allow to quantify for linear photonic crystal waveguides the theoretical limit of intrinsic diffraction losses, which assumes ideal structures without disorder, roughness on non-ideal hole effects.

Acknowledgements The author is grateful to M. Agio for several helpful discussions. This work was supported in part by INFM PRA project "PHOTONIC" and by MIUR through Cofin 2000 program.

\section{References}

[1] E. Yablonovitch, Phys. Rev. Lett. 58, 2059 (1987).

[2] S. John, Phys. Rev. Lett. 58, 2486 (1987).

[3] J.D. Joannopoulos, R.D. Meade, and J.N. Winn, Photonic Crystals - Molding the Flow of Light, Princeton University Press, 1995.

[4] K. SaKoda, Optical Properties of Photonic Crystals, Springer, Berlin 2001.

[5] T.F. Krauss, R.M. De La Rue, and S. Brand, Nature 383, 699 (1996).

[6] H. Benisty, C. Weisbuch, D. Labilloy, M. Rattier, C.J.M. Smith, T.F. Krauss, R.M. De La Rue, R. Houdré, U. Oesterle, C. Jouanin, and D. Cassagne, J. Lightwave Technol. 17, 2063 (1999).

[7] S.G. Johnson, S. Fan, P.R. Villeneuve, J.D. Joannopoulos, and L.A. Kolodziejski, Phys. Rev. B 60, 5751 (1999).

[8] V.N. Astratov, D.M. Whittaker, I.S. Culshaw, R.M. Stevenson, M.S. Skolnick, T.F. Krauss, and R.M. De La Rue, Phys. Rev. B 60, R16225 (1999).

[9] D.M. WhitTaKer and I.S. Culshaw, Phys. Rev. B 60, 2610 (1999).

[10] H. Benisty, D. Labilloy, C. Weisbuch, C.J.M. Smith, T.F. Krauss, D. Cassagne, A. Béraud, and C. Jouanin, Appl. Phys. Lett. 76, 532 (2000).

[11] M. Loncar, D. Nedeljkovic, T. Doll, J. Vuckovic, A. Scherer, and T.P. Pearsall, Appl. Phys. Lett. 77, 1937 (2000).

[12] E. Chow, S.Y. Lin, S.G. Johnson, P.R. Villeneuve, J.D. Joannopoulos, J.R. Wendt, G.A. Vawter, W. ZubrZYCKI, H. Hou, and A. Alleman, Nature 407, 983 (2000).

[13] Ph. Lalanne and H. Benisty, J. Appl. Phys. 89, 1512 (2001).

[14] N. Kawai, K. Inoue, N. Carlsson, N. Ikeda, Y. Sugimoto, K. Asakawa, and T. Takemori, Phys. Rev. Lett. 86, 2289 (2001).

[15] T. Ochial and K. Sakoda, Phys. Rev. B 63, 125107 (2001).

[16] T. Ochial and K. SAKoda, Phys. Rev. B 64, 045108 (2001).

[17] S.G. Tikhodeev, A.L. Yablonskit, E.A. Muljarov, N.A. Gippius, and T. Ishihara, Phys. Rev. B 66, 045102 (2002).

[18] M. Le Vassor D'Yerville, E. Centeno, D. Cassagne, and J.P. Albert, to be published.

[19] L.C. ANDREANi and M. Agio, IEEE J. Quantum Electron. 38, 891 (2002).

[20] M. Galli, M. Agio, L.C. Andreani, L. Atzeni, D. Bajoni, G. Guizzetti, L. Businaro, E. Di Fabrizio, F. Romanato, and A. Passaseo, Eur. Phys. J. B 27, 79 (2002).

[21] M. Patrini, M. Galli, F. Marabelli, M. Agio, L.C. Andreani, D. Peyrade, and Y. Chen, IEeE J. Quantum Electron. 38, 885 (2002). 